\title{
Design Variable Cross Section Roll-forming Part Using Bezier Curve
}

\author{
X. Huang, D. Zhao and J. Wang \\ North China University of Technology \\ School of Mechanical and Materials Engineering \\ marchbupt@126.com
}

\begin{abstract}
This paper proposes a continuous stitching Bezier curve interpolation algorithm to assure the bending curve of a variable cross-section roll forming parts is smooth and continuous. By proposed algorithm the recursive equation is derived into solving the problem of Bezier curve control points. In order to make the bending curve smooth at each interpolation point, the continuity condition of two combined Bezier curve in Euclidean space curves is analyzed. With the proposed method, the accuracy and $G^{2}$ continuity of the whole bending curve are obtained. Due to abandoning the traditional method which utilized straightly line segments and arc segments to construct the bending curve, the propose method can improve the design accuracy and realize the parametric design. The simulation using Mathematics soft demonstrates the feasibility of part design based on the proposed method. The research provides the theoretical basis for the control system of the variable cross section roll-forming part design.
\end{abstract}

Keywords: Bezier curve, Variable cross section roll-forming, Parametric design

\section{Introduction}

The variable cross-section roll forming is a novel gradually-forming technology, which is developed from the traditional fixed section roll forming technology [1]. Compared with the traditional roll forming technology, there are some advantages in improving the processing quality, reducing processing costs and reducing the processing time. The variable cross-section roll forming has become an effective method to make the car front bumper, B column reinforced plate and other complicated metal parts. In the process of cross section roll-forming, the curve scanned by roller, named bending curve, determines directly the feed speed, the accuracy of processing and the stability of the machine tool. Therefore, the design of the bending curve is the research focus of the variable cross section roll forming parts. In the process of cross section roll forming, the bending curve is usually composed by linear and circular segments. The continuity of bending curve is G1 continuity. That means that the tangential direction of the curve is same at the splicing point, but the curvature of a curve is sudden changed which will cause the vibration of the mechanical machining.

To compensate for the insufficient of linear interpolation and circular interpolation, scholars have carried out a great deal of studies on smooth continuous curve. CAI etc. [2] used B-spline interpolation method to describe the shape of the flexible roll axis. Although the cubic B-spline curve control points can be obtained from the tabulated curve, the computation time of control points is very long when the control points are dense. What's more, the control points need to calculate once again when one of the control points has been changed. ZHOU etc. [3-6] applied the Bezier curve fitting on the blade modeled lines. This method, which has shown distinct advantages over other methods, not only eliminated the approximation of cylindrical modeled lines by one or several arcs, but also improved the design accuracy. CHU etc. [7] proposed a way of application of the 
Bezier curve to design the cam profiles of the indexing cam mechanisms. The method is very valuable to enhance the dynamic characters and improve the precision locking of the indexing mechanisms. ZHAO [8] presented a skull recognition algorithm by quadratic rational Bezier curve fitting which accurately described the feature of skull edge. WANG [9] proposed an algorithm for fitting the cavity lateral surface using fitting algorithm of blending rational Bezier surface. According to the experimental, the fitting algorithm can control the machining precision, improve the surface quality. WANG [10] proposed a Bezier curve interpolation algorithm applied in CNC system. The method of smooth and continuous 3th Bezier curve provides a feasible scheme for curve modeling.

To obtain a smooth and practical bending curve for the variable cross-section roll forming products, this paper proposes a cubic Bezier curve interpolation method. This method derives the recursive equation to solve Bezier curve control points. The G2 continuity conditions of two random degree adjacent rational Bezier curves are also studied. Finally, the cubic Bezier bending curves is obtained with the G2 continuity condition.

\section{Bezier Curve}

Bezier curve is a kind of design method based on approximations of parametric curves and surfaces. Bezier curve combines the approximating function with geometric representation, and can help designers to realize easily the relationship between design of the curve and condition given in engineering and change easily the shape of the curve by controlling the input parameters. Bezier curve with good geometric properties is widely used in the CAD/CAM modeling technology.

\subsection{Basic Definition}

Bezier curve is a parametric polynomial curve determined uniquely by a set of vertices of the control polygon. Given a set of $n+1$ spatial point $P_{0}, P_{1}, P_{2}, \ldots, P_{n}$, the definition of Bezier curve is as the following

$$
C(t)=\sum_{k=0}^{n} P_{k} B_{k, n}(t) \quad t \in[0,1]
$$

where, $B_{k, n}(t)$ is the Bernstein basis function

$$
B_{k, n}(t)=C_{n}^{k} t^{k}(1-t)^{n-k}=\frac{n !}{k !(n-k) !} t^{k}(1-t)^{n-k} \quad t \in[0,1], k=0,1, \cdots, n
$$

\subsection{The Properties of the Endpoint}

The starting and ending points of Bezier curve are coincided with the starting and ending points of control polygon respectively.

When $t=0$, the equation (1) can be written as

$$
C(0)=B_{0, n}(0) \cdot P_{0}+B_{1, n}(0) \cdot P_{1}+\cdots=P_{0}
$$

When $t=1$, the equation (1) can be written as

$$
C(1)=B_{0, n}(1) \cdot P_{0}+\ldots+B_{n, n}(1) \cdot P_{n}=P_{n}
$$

The first order derivative of the Bezier curve can be derived from (1) and written as

$$
\begin{aligned}
& C^{\prime}(t)=\sum_{k=0}^{n} P_{k} B_{k, n}^{\prime}(t) \quad t \in[0,1] \\
& =n \sum_{k=1}^{n}\left(P_{k}-P_{k-1}\right) B_{k-1, n-1}(t) \\
& =n\left(\left(P_{1}-P_{0}\right) B_{0, n-1}(t)+\left(P_{2}-P_{1}\right) B_{1, n-1}(t)+\ldots+\left(P_{n}-P_{n-1}\right) B_{n-1, n-1}(t)\right) \text { At the beginning point, } \\
& t=0, B_{0, n-1}(0)=1 \text {, the remaining parameters are } 0, \text { so }
\end{aligned}
$$




$$
C^{\prime}(t)=n\left(P_{1}-P_{0}\right)
$$

At the ending point, $t=1, B_{n-1, n-1}(0)=1$, the remaining parameters are 0 , so

$$
C^{\prime}(t)=n\left(P_{n}-P_{n-1}\right)
$$

It means that the first derivative of Bezier curve at the endpoint is only associated with two adjacent control points and the tangential direction of the Bezier curve is the direction of point's line.

The second order derivative of the Bezier curve can be derived from (1) and written as

$$
C^{\prime \prime}(t)=n(n-1) \sum_{k=0}^{n}\left(P_{k+2}-2 P_{k+1}+P_{k}\right) B_{k, n-1}(t)
$$

At the beginning point $t=0$, the second order derivative of Bezier curve is

$$
C^{\prime \prime}(0)=n(n-1)\left(P_{2}-2 P_{1}+P_{0}\right)
$$

At the ending point $t=1$, the second order derivative of Bezier curve is

$$
C^{\prime \prime}(1)=n(n-1)\left(P_{n}-2 P_{n-1}+P_{n-2}\right)
$$

where, $C^{\prime \prime}(0), C^{\prime \prime}(1)$ are the second order derivative of the Bezier curve at the beginning and ending points respectively. So the second order derivative of Bezier curve at the endpoint is only associated with three adjacent control points. That means the derivation of order $\mathrm{r}$ is only associated with $n+1$ adjacent control points and unrelated to further point.

\section{Continuity of Bezier Interpolation}

\subsection{Cure Matching of Bezier Curve}

In the section, the Bezier function is used to describe the bending curve in the variable cross-section roll forming. In order to make the curve smoothly connected at each interpolation point, continuity condition of two Bezier curves should be derived.

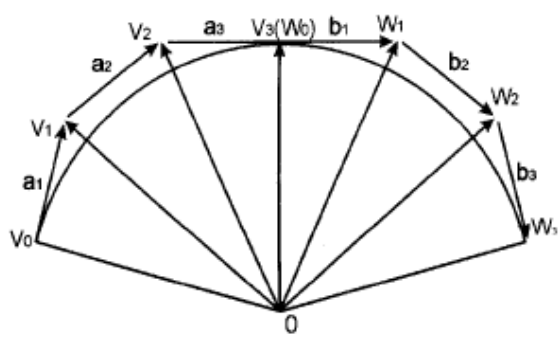

Figure 1. Cure Matching of two Bezier Curve

Combining the endpoint properties of Bezier curve with the cure matching of two Bezier curve is shown in Figure 1. The relationship between the adjacent curve's control points can be written as following

$$
\left\{\begin{array}{c}
W_{1}=2 V_{3}-V_{2} \\
W_{2}=2 V_{3}-3 V_{2}+V_{1}+W_{1}
\end{array}\right.
$$

When the Bezier function is used to fit the bending curve of the variable cross-section roll forming, the bending curve satisfies the equation (2). The first derivative and second derivative of the curve at each points is uniformly continuous which means the velocity and acceleration is continuous.

\subsection{Recurrence Formula of the Control Point}

Given a set of curve interpolation points $P_{0}, P_{1}, P_{2}, \ldots, P_{n}$ each curve between two adjacent can be fitted by Bezier curves. Setting the $i^{\text {th }}$ curve is Bezier curve, $P_{i-1}, P_{i}$ are the 
interpolation points and $P_{i-1}, P_{i-1}^{(1)}, P_{i}^{(2)}, P_{i}$ are four control points. The $i^{\text {th }}$ cubic Bezier curve is shown as the following

$$
P_{i}^{(u)}=\left[\begin{array}{llll}
u^{3} & \mathrm{u}^{2} & \mathrm{u} & 1
\end{array}\right]\left[\begin{array}{cccc}
-1 & 3 & -3 & 1 \\
3 & -6 & 3 & 0 \\
-3 & 3 & 0 & 0 \\
1 & 0 & 0 & 0
\end{array}\right]\left[\begin{array}{c}
P_{i-1} \\
P_{i}^{(1)} \\
P_{i}^{(2)} \\
P_{i}
\end{array}\right]
$$

where,

$$
\left\{\begin{array}{c}
P_{i}^{(1)}=2 P_{i}-P_{i-1}^{(2)} \\
P_{i}^{(2)}=2 P_{i}-3 P_{i-1}^{(2)}-P_{i-1}^{(1)}+P_{i}^{(1)}
\end{array}\right.
$$

The equation (4) is the recurrence formula of the Bezier control points. With the Boundary Conditions and recurrence formula of the Bezier control points, we can generate the smoothly connected Bezier curve.

\subsection{Example of Cure Matching of Bezier Curve}

Given four interpolation points in space, we can design a smooth and $G^{2}$ continuous Bezier curves. By the boundary conditions and recurrence formula of control point, the calculated Bezier curve control points are shown in Table 1.

\section{Table 1. Bezier Curve Control Points}

\begin{tabular}{ccccc}
\hline Bezier control points & $b[i]$ & $b[i+1]$ & $b[i+2]$ & $b[i+3]$ \\
\hline$i=0$ & $\{2,80\}$ & $\{10 / 3,220 / 3\}$ & $\{20 / 3,110 / 3\}$ & $\{8,30\}$ \\
$i=1$ & $\{8,30\}$ & $\{28 / 3,70 / 3\}$ & $\{14,140 / 3\}$ & $\{15,70\}$ \\
$i=2$ & $\{15,70\}$ & $\{16,280 / 3\}$ & $\{52 / 3,350 / 3\}$ & $\{23,50\}$ \\
\hline
\end{tabular}

The Bezier curve can be obtained by the Bezier curve control points in Table 1, showed as following.

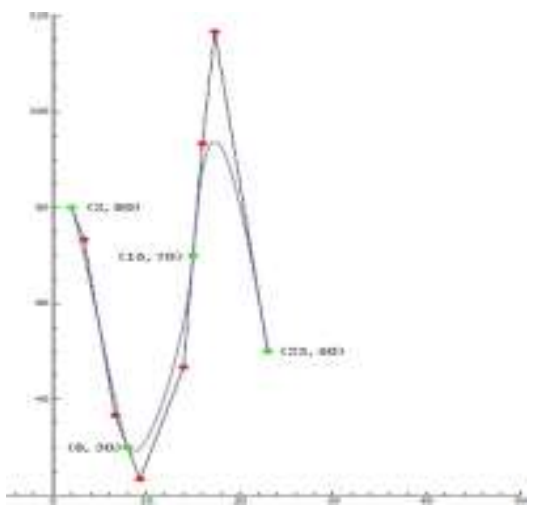

Figure 2. The $\mathrm{G}^{2}$ Continuous Cubic Bezier Curves

The green points in coordinate are interpolation points and the red points are intermediate Bezier control points generated by the equation (4). This method makes the first derivative and second derivative of the curve continuous at the interpolation points. 


\section{Design of Bending Curve and Roll Forming Part}

\subsection{Bending Curve Design}

In the process of the variable cross-section roll forming part design, Bezier curve is used instead of circular curve. In this method, a smooth and piecewise $G^{2}$ continuous cubic Bezier curves is designed, as shown in Figure 3.

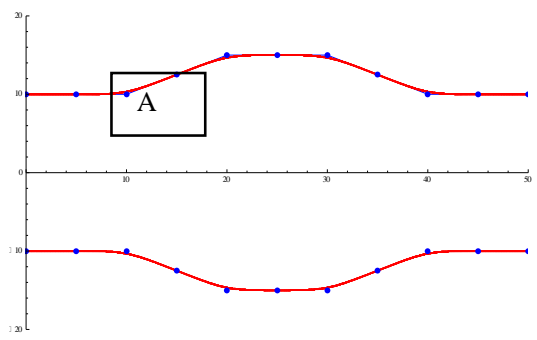

\section{Figure 3. The Cubic Piecewise Bezier Curves}

For a clearer contrast stitching curves in position A, the local contrast diagram of $\mathrm{A}$ are given, as shown in Figure 4

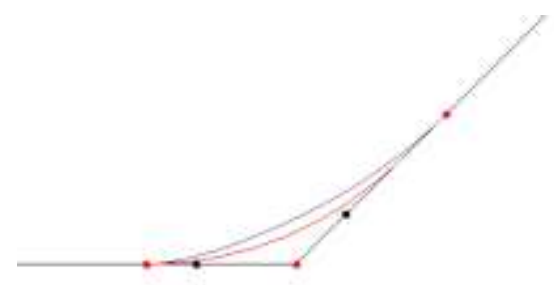

Figure 4. The Location Contrast Diagram of $A$

In Figure 4, the red line is Bezier continuous interpolation curve, the blue line is the circular arc curve, and two black points are Bezier control points. It was obvious that curvature of Bezier curve is smaller at the endpoint in Figure 4. Therefore, at the interpolation points, Bezier splicing curve is smoother than the stitching arc curve

In the design process of three-dimensional roll forming bending curve, bending curve which is the Bezier curve and Bezier stitching curve that can effectively improve the machine vibration in the splicing point.

\subsection{Roll Forming Part Design}

Combining the roller shape of variable cross-section roll forming with bending curve design by Bezier curve shown in Figure 3, the simulation diagram of the variable crosssection roll forming parts is generated based on the Mathematica software, as shown Figure 5 . 


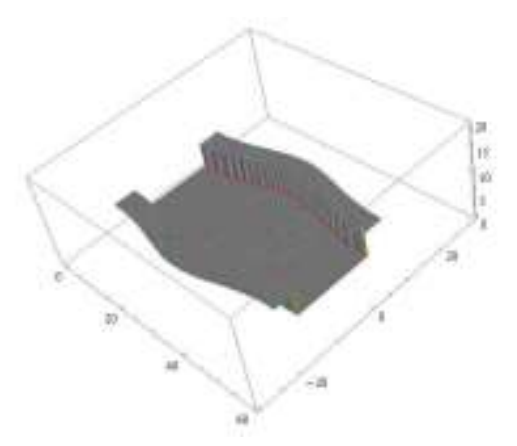

Figure 5. Simulation Diagram of Part

\section{Conclusions}

This paper proposes a method for application of the Bezier curve to design the part of variable cross-section of the roll forming. A parametric model of bending curve based on the third- order Bezier curve. Bending curve using the method of the third-order Bezier curve interpolation is smoother the curve splicing by linear and circular curves. Through experiment and analysis of mathematical model, the feasibility of part design based on Bezier curve is verified. The research results provide theoretical basis for Interpolation algorithm of the control system of the 3D roll forming production line.

\section{Acknowledgements}

This work is partially supported by National Science Foundation of PR China (51105003).

\section{References}

[1] Z. Ai, J. Liu and F. Han, "Research Improvement of Variable Section Flexible Roll Forming Technology at Home and Aboard", Materials \& Manufacturing Processes, vol. 19, (2010), pp. 34-36.

[2] Z. Cai, M. Li and Y. Lan, "Shape control of 3-dimensional curved surface part in continuous forming", Journal of Jilin University (Engineering and Technology Edition), vol. 41, (2011), pp. 978-982.

[3] W. Zhou, R. Wang and Z. Shen, "Application of Bezier Curve on the 3D Blade Profiling of Bottom Hole Intensifier Centrifugal Pump”, Drilling \& Production Technology, vol. 31, (2008), pp. 84-86.

[4] W. Zhuang, X. Fang and P. Wang, "The design of interactive program for three-dimensional turbine blades generation", Machinery Design \&Manufacture, vol. 10, (2005), pp. 53-55.

[5] W. Zou, S. Hu and J. Zhao, "Analysis for Effect of Blade Outlet Angle on the Performance of Pump Based on Bezier Curve". Chinese Quarterly of Mechanics, vol. 33, (2012), pp. 130-133.

[6] X. Hu, Q. Liu and Y. Liu, "The Application of Using Bezier Curve to Design Turbine Blade", Oil Field Equipment, vol. 36, (2007), pp. 1-3.

[7] F. Chu, C. Meng and Y. Yang, "Application of the Bezier Curve to Design the Cam Profiles of the Indexing Cam Mechanisms", Mechanical Science and Technology for Aerospace Engineering, vol. 18, (1999), pp. 551-553.

[8] W. Zhao, X. Xie and F. Yang, "On Skull Recognition of Quadratic Rational Bezier Curve Fitting". Journal of Biomedical Engineering. vol. 25, (2008), pp. 280-284

[9] Q. Wang, Q. Liu and X. Liang, "Cavity surface construction of type tread pattern mold and its finish machining", Journal of Zhejiang University (Engineering Science). vol. 40, (2006), pp. 399-403

[10] Y. Wang, G. Yang, "An Application of Bezier Curve Interpolation Algorithm in CNC Systems", Mechanical \& Electrical Engineering Magazine, vol. 17, (2000), pp. 46-47. 